\title{
Incorporating chemical signalling factors into cell-based models of growing epithelial tissues
}

\author{
Aaron M. Smith · Ruth E. Baker • David Kay • \\ Philip K. Maini
}

Received: 21 July 2011 / Published online: 7 September 2011

(C) Springer-Verlag 2011

\begin{abstract}
In this paper we present a comprehensive computational framework within which the effects of chemical signalling factors on growing epithelial tissues can be studied. The method incorporates a vertex-based cell model, in conjunction with a solver for the governing chemical equations. The vertex model provides a natural mesh for the finite element method (FEM), with node movements determined by force laws. The arbitrary Lagrangian-Eulerian formulation is adopted to account for domain movement between iterations. The effects of cell proliferation and junctional rearrangements on the mesh are also examined. By implementing refinements of the mesh we show that the finite element (FE) approximation converges towards an accurate numerical solution. The potential utility of the system is demonstrated in the context of Decapentaplegic (Dpp), a morphogen which plays a crucial role in development of the Drosophila imaginal wing disc. Despite the presence of a Dpp gradient, growth is uniform across the wing disc. We make the growth rate of cells dependent on Dpp concentration and show that the number of proliferation events increases in regions of high concentration. This allows hypotheses regarding mechanisms of growth control to be rigorously tested. The method we describe may be adapted to a range of potential application areas, and to other cell-based models with designated node movements, to accurately probe the role of morphogens in epithelial tissues.
\end{abstract}

\footnotetext{
A. M. Smith $(\varangle) \cdot$ R. E. Baker · P. K. Maini

Centre for Mathematical Biology, Mathematical Institute, 24-29 St. Giles',

Oxford OX1 3LB, UK

e-mail: smitha1@maths.ox.ac.uk

P. K. Maini

Department of Biochemistry, Oxford Centre for Integrative Systems Biology, South Parks Road, Oxford OX1 3QU, UK

D. Kay

Department of Computer Science, Wolfson Building, Parks Road, Oxford OX1 3QD, UK
} 
Keywords Tissue growth · Diffusion - Vertex models ·

Arbitrary Lagrangian-Eulerian · Finite elements · Drosophila imaginal wing disc

Mathematics Subject Classification (2000) $\quad 92 \mathrm{C} 15 \cdot 92-08 \cdot 35 \mathrm{Q} 92$

\section{Introduction}

Several recent studies have examined the effects of cell proliferation, rearrangements and mechanical forces on tissue growth and epithelial packing (Farhadifar et al. 2007; Rauzi et al. 2008; Bittig et al. 2008; Aegerter-Wilmsen et al. 2010). Concurrently, there has been much interest in the role played by chemical signalling molecules known as morphogens, which form gradients in many developmental systems in order to coordinate tissue patterning and stimulate growth (Affolter and Basler 2007). To date, however, there has been little attempt to combine these strands into a single coherent modelling framework.

Aegerter-Wilmsen et al. (2007) used a tissue-level model to show that net growth of the Drosophila imaginal wing disc can be regulated by a combination of morphogen gradients and mechanical forces. In their model, growth factors initially stimulate growth in the middle of the wing disc, leading to a stretching of the lateral regions. This, in turn, induces growth in those areas via mechanical feedback, which eventually causes compression back at the center of the disc, inhibiting growth. Their model crucially accounts for the termination of growth when the wing disc reaches a certain size. More recently, the same authors (Aegerter-Wilmsen et al. 2010) used a vertex model and showed that, by including mechanical feedback as a regulator for cellular growth, they could faithfully reproduce the experimentally observed polygon distribution of the wing disc tissue. Hufnagel et al. (2007), meanwhile, also use mechanical feedback within a vertex model, with cell-growth rate dependent on the height of each cell, which acts as an indicator of the stress it is under. Growth can only occur, however, when a minimum threshold of the morphogen Decapentaplegic (Dpp) is exceeded, with the Dpp gradient imposed as a simple radial function, exponentially decaying with a constant characteristic length.

Wartlick et al. (2011) implemented morphogen dynamics in a vertex model by assigning molecule numbers for two morphogens to each cell and solving a system of coupled differential equations representing production, degradation, and transport. They showed experimentally that the concentration and signalling gradients of Dpp in the Drosophila wing disc scale with tissue size during development, and that an increase in signalling levels by $50 \%$ induces cell division. By implementing these observations in the vertex model they showed that temporal changes in Dpp levels could be an important mechanism in regulating growth.

Vertex models have been used in several other recent studies of growth. Landsberg et al. (2009), for example, showed that the antero-posterior compartment boundary of the Drosophila wing disc can be maintained by a 2.5 -fold increase in tension at cell edges near the boundary. They were also able to confirm experimentally that this increase in tension is realistic. Farhadifar et al. (2007), meanwhile, examined the effects of proliferation, and found regions of parameter space where their vertex 
model accurately represents the packing geometry of the growing wing disc. Rauzi et al. (2008) used a vertex model to show that anisotropy of cortical tension can drive tissue elongation. This was achieved by including an angle dependence in the tension force (see Sect. 2.1).

Here we describe a novel method for dynamically incorporating chemical signalling into vertex models, using an arbitrary Lagrangian-Eulerian (ALE) formulation in conjunction with the finite element (FE) method (FEM) to solve equations for chemical concentration. Some of the models described above include basic representations of morphogen gradients, but none feature a complete dynamic description of the spreading of chemical signalling factors. The method described in this paper is the first complete framework that models the mechanical properties of cells along with an accurate, realistic description of morphogen concentration. It also exhibits key physical properties such as conservation of mass, and is able to account for the movement and growth of the domain as well as important cellular processes such as proliferation and junctional rearrangements. The method has applications in many biological systems as it can be adapted to any process in which a chemical spreading across an epithelium interacts with the mechanical properties of a cell.

Section 2 begins by describing the vertex model, including descriptions of the force laws, equations of motion, junctional rearrangements, cell growth and proliferation. Section 3 then goes on to describe the morphogen spreading model, in particular the ALE formulation and FE approximation to the solution. Section 4 examines the convergence properties of the FE approximation, while Sect. 5 discusses the length of time taken to form and solve the governing matrix system of equations. Methods for dealing with mitosis and junctional rearrangements in the FE scheme are described in Sect. 6. In Sect. 7 the method is applied to Dpp in the Drosophila wing disc, and some initial results are presented, before the summary and discussion in Sect. 8.

\section{The vertex model}

Two-dimensional (2D) networks of polygons have long been known to successfully capture the packing geometry of epithelial tissues (Honda 1978). Shape, size, and movement of cells emerge from the application of forces to polygon vertices (Weliky and Oster 1990; Nagai and Honda 2001). Typically, forces represent cell-cell adhesion, actin-myosin contractility, elasticity, pressure, and protrusion. These are dependent on the basic properties of each cell, such as perimeter, area, height, and deformation. As well as basic force laws, junctional rearrangements, growth, and proliferation can be included. The model described here consists of two types of forces, which act in the directions shown in Fig. 1a.

\subsection{Tension forces}

In this model, stress on each cell edge manifests as a tension force on the vertices at the ends of the edge. The first component of this force is a line tension, dependent on the edge length, which represents effects of cell-cell adhesion and actin-myosin 


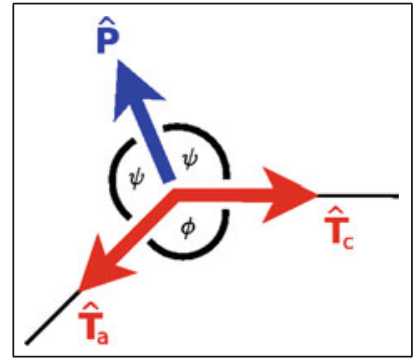

(a)

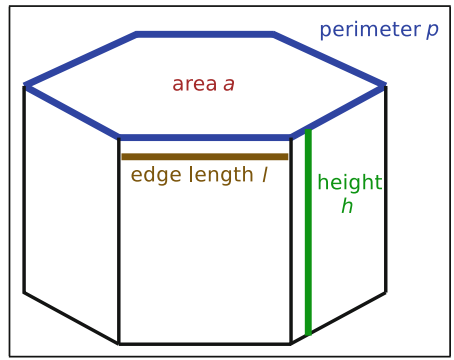

(b)

Fig. 1 a Direction of tension $(\hat{\mathbf{T}})$ and pressure $(\hat{\mathbf{P}})$ forces acting on vertices in the model. Tension forces, representing cell-cell adhesion and actin-myosin contractility, act along cell edges in clockwise $\left(\hat{\mathbf{T}}_{c}\right)$ and anti-clockwise $\left(\hat{\mathbf{T}}_{a}\right)$ directions. Pressure forces, representing cell elasticity, elongation and deformation, bisect the angle $\phi$ between two edges. b Three-dimensional schematic of an epithelial cell, showing four key quantities used in the model; perimeter, $p$, area, $a$, height, $h$, and edge length, $l$

contractility. Stronger adhesion or reduced contractility leads to reduced stress on the edge, and thus lower tension and larger edge lengths. The second component, meanwhile, depends on the length of the cell perimeter and represents contractility of the actin-myosin ring.

Within a given cell each vertex belongs to two edges and experiences forces in the direction of the two tension unit vectors shown in Fig. 1a. The contribution from a cell to the tension on a vertex is given by

$$
\mathbf{T}=C_{L}\left(l_{c} \hat{\mathbf{T}}_{c}+l_{a} \hat{\mathbf{T}}_{a}\right)+C_{P}\left(\hat{\mathbf{T}}_{c}+\hat{\mathbf{T}}_{a}\right) p,
$$

where $C_{L}$ and $C_{P}$ are constants related to the line tension and perimeter force, respectively, $l_{c}$ and $l_{a}$ are the lengths of the clockwise and anti-clockwise edges, $\hat{\mathbf{T}}_{c}$ and $\hat{\mathbf{T}}_{a}$ are unit vectors in the direction of the clockwise and anti-clockwise vertices, and $p$ is the total length of cell perimeter (see Fig. 1b). The constants $C_{L}$ and $C_{P}$ have units of force over distance (e.g. $\mathrm{N} \mathrm{m}^{-1}$ ). The tension force acts to decrease the length of edges and perimeters. Larger edge lengths and perimeters generate larger tension forces, which act to move neighbouring vertices closer to each other, thus shrinking local edge lengths.

\subsection{Pressure forces}

The pressure force, meanwhile, is dependent on cell area, height-to-area ratio, and local deformation. It is given by

$$
\mathbf{P}=\left[C_{A} \frac{\left|a_{t}-a\right|^{n_{1}+1}}{\left(a_{t}-a\right)}+C_{H} H+C_{D} \frac{|\phi-\theta|^{n_{2}+1}}{(\phi-\theta)}\right] \hat{\mathbf{P}}
$$

where $C_{A}, C_{H}$, and $C_{D}$ are constants associated with the three components, $a$ is the cell area, $a_{t}$ is a 'target' area, $H$ is the height-to-area ratio, $\theta$ is the average internal 
angle of the cell $(\theta=\pi(s-2) / s$ for an $s$-sided polygon), $\phi$ is the internal angle at the current vertex, $n_{1}$ and $n_{2}$ are integer values, and $\hat{\mathbf{P}}$ is a unit vector in the direction of the pressure force (see Fig. 1a). The first and third terms are written in this way to ensure that the sign of the force is correct regardless of whether $n_{1}$ and $n_{2}$ are even or odd.

The first term represents the elasticity of the cell. $C_{A}$, which has units of force per distance to the power $2 n_{1}$ (e.g. $\mathrm{N} \mathrm{m}^{-2 n_{1}}$ ), is the elastic coefficient. The second term represents the effect of a cell opposing being squeezed or squashed and elongating in the direction normal to the surface. Each cell is assigned a volume, drawn from a normal distribution, which can be allowed to grow over time (see Sect. 2.6). Knowing the volume and area of every cell, heights can then be calculated. The constant $C_{H}$ has units of force times distance.

The third term in (2) represents the reaction of the internal components of the cell to being subjected to an external force. In vivo, concave cells arise occasionally when they are subjected to significant pressure, but will not stay in this configuration for very long. This is not a natural state for cells to be in, and therefore we include a force in the model designed to keep cells as near to their regular shape as possible. The constant in this case, $C_{D}$, has units of force per angle to the power $n_{2}$ (e.g. $\mathrm{N} \mathrm{rad}^{-n_{2}}$ ).

Figure $1 \mathrm{~b}$ shows a three-dimensional (3D) epithelial cell, demonstrating the key quantities used in the model. In this model forces act on the top (apical) surface. The model is not fully $3 \mathrm{D}$, in the sense that we do not take account of the fact that apical surfaces of neighbouring cells may not be at the same level. We do, however, consider cells to be $3 \mathrm{D}$ objects with both volume and height.

\subsection{Boundary force}

An extra force is implemented to ensure integrity of the tissue is maintained and edges of non-adjacent cells are unable to overlap on the boundary. This force only applies to vertices on the boundary, which can be identified by the fact that they belong to a maximum of two cells, and takes the form

$$
\mathbf{B}=C_{B 1} \frac{\left|\phi_{B}-\theta_{B}\right|^{n_{3}+1}}{\left(\phi_{B}-\theta_{B}\right)} \hat{\mathbf{P}}_{B}+C_{B 2}\left(\frac{\left|l_{B c}-L\right|^{n_{4}+1}}{\left(l_{B c}-L\right)} \hat{\mathbf{T}}_{B c}+\frac{\left|l_{B a}-L\right|^{n_{4}+1}}{\left(l_{B a}-L\right)} \hat{\mathbf{T}}_{B a}\right),
$$

where $C_{B 1}$ and $C_{B 2}$ are the boundary force constants, $\phi_{B}$ is the internal angle at the boundary vertex, $\theta_{B}$ is the ideal internal angle, given by $\theta_{B}=\pi\left(N_{B}-2\right) / N_{B}$ for $N_{B}$ boundary nodes, $\hat{\mathbf{P}}_{B}, \hat{\mathbf{T}}_{B C}$ and $\hat{\mathbf{T}}_{B a}$ are unit vectors in the direction of the pressure- and tension-like forces, $l_{B C}$ and $l_{B a}$ are the lengths of the clockwise and anti-clockwise edges, respectively, $L$ is the mean edge length, and $n_{3}$ and $n_{4}$ are integers.

This force tends to make the boundary smooth and circular, and keep boundary edges from shrinking. Junctional rearrangements (Sect. 2.5) are unable to occur on the boundary, so it is important that edges do not become very small, hence there is a difference between the edge-dependent force here and that in the tension Eq. (1). 
The angle-dependent force is similar to that in the pressure-force Eq. (2), which keeps cells as close to regular as possible.

\subsection{Equations of motion}

In generating equations of motion, it is assumed that viscous forces dominate and inertial considerations may be neglected (Odell et al. 1981). For a vertex $i$ the equation of motion is given by

$$
\mu_{i} \frac{\mathrm{d} \mathbf{x}_{i}}{\mathrm{~d} t}=\mathbf{F}_{i}
$$

where $\mu_{i}$ is the viscous coefficient, $\mathbf{x}_{i}$ is the vertex position, and $\mathbf{F}_{i}$ is the sum of all forces acting on the vertex. We define a discretisation in time over the range $(0, T]$, given by $\cup_{n=1}^{n=N_{T}}\left(t^{n-1}, t^{n}\right.$ ], where $T$ is the final time, $N_{T}$ is the number of time-points, and $t^{n}-t^{n-1}=\Delta t$ for all $n$. The system can be solved iteratively at each time-point using the forward Euler method as follows:

$$
\mathbf{x}_{i}^{n+1}=\mathbf{x}_{i}^{n}+\frac{\Delta t}{\mu_{i}} \mathbf{F}_{i}^{n}
$$

\subsection{Junctional rearrangements}

In addition to the force laws described above, an elementary rearrangement of vertices known as a T1 transition is included in the model. Junctional rearrangements occur between neighbouring vertices that fall below a certain threshold distance. This type of transition has been used by other authors in previous vertex models (Weliky and Oster 1990; Farhadifar et al. 2007). Figure 2 explains a T1 transition diagrammatically. Vertices A and B have moved closer together than the threshold distance, and rearrangement begins (a). Vertices $\mathrm{C}$ and $\mathrm{D}$ are created on a line that bisects the line $\mathrm{AB}$ perpendicularly. Cell 1 then reconnects to vertex $\mathrm{C}$, cell 2 to vertex $\mathrm{D}$, and cells 3 and 4 share both vertices $\mathrm{C}$ and $\mathrm{D}$ (b).

\subsection{Cell growth and proliferation}

To accommodate a method allowing cells to grow and subsequently divide, each cell $k$ is given a growth speed, $g_{k}$, drawn from a truncated normal distribution. Growth is then implemented logistically over time according to

$$
v_{k}^{n+1}=v_{k}^{n}\left(1+g_{k} \Delta t\left(1-\frac{v_{k}^{n}}{V_{T}}\right)\right),
$$

where $v_{k}^{n}$ is the volume of cell $k$ at time $t^{n}$ and $V_{T}$ is a target volume.

To implement cell division, firstly an angle of mitosis is chosen. This can simply be drawn from a uniform distribution, or biased in a certain direction if desired to 


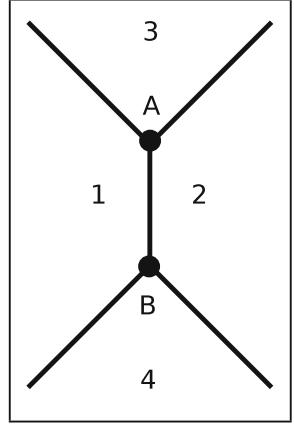

(a)

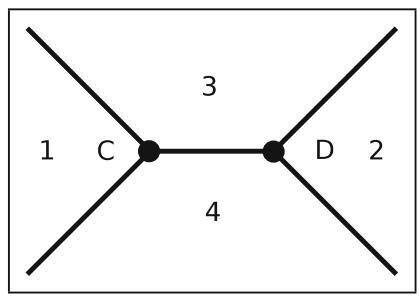

(b)

Fig. 2 A T1 transition for two close vertices. a Initially cells 1 and 2 share edge AB, which separates cells 3 and 4 . b After the rearrangement cells 3 and 4 share edge CD, separating cells 1 and 2

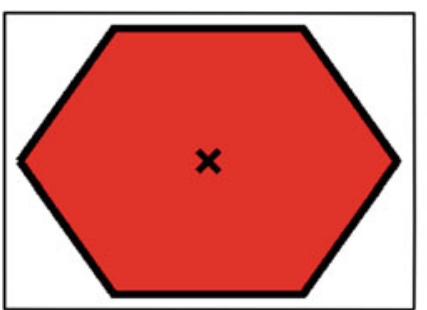

(a)

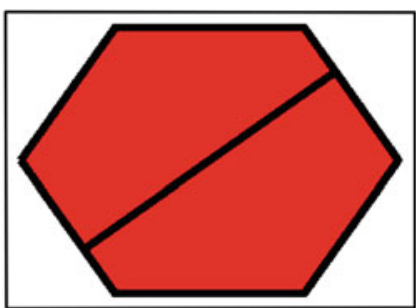

(b)

Fig. 3 Cell division in the vertex model. a The initial cell, with a cross at the center. b A straight line is drawn through the cell center at an angle drawn from a uniform distribution, and two new vertices are formed where the line meets cell edges

investigate directed proliferation. A straight line is then drawn, passing through the centroid of the cell at the prescribed angle. Two new vertices are created at the points where the line intercepts cell edges. Figure 3 demonstrates this process: (a) shows the initial cell that has been chosen to divide, with the cross indicating the centroid of the cell. In (b) the cell has divided into two daughter cells.

Cells are allowed to divide with probability based on the ratio of their current volume to the target volume. This ensures that cells close to the target volume are likely to divide, whereas those which are much smaller have very little chance of proliferating.

We now have a complete physical model of cell behaviour. We have seen the force laws, junctional rearrangements, as well as cell growth and proliferation. In the next section we will develop a model of chemical signalling factors. We will see how the chemical concentration can feedback into the equation for cell growth (5), which is currently independent of any morphogen concentration. 


\section{Modelling morphogen concentrations}

\subsection{Diffusion equation and ALE formulation}

Kicheva et al. (2007) stated that, regardless of the actual mechanism of transport, morphogen spreading can be captured by the physics of molecules produced in a central strip of cells, which spread non-directionally and are degraded at some rate. The concentration of morphogen across a domain can therefore be represented by a diffusion equation with source term, of the form

$$
\frac{\partial c}{\partial t}-\nabla \cdot(D \nabla c)=f \quad \text { in } \Omega(t)
$$

where $c(\mathbf{x}, t)$ is the concentration at point $\mathbf{x}$ on domain $\Omega(t)$ at time $t, D(\mathbf{x}, t)$ is the diffusion coefficient, and $f(\mathbf{x}, t)$ is some known source/degradation function. The initial condition is $c(\mathbf{x}, 0)=c_{0}(\mathbf{x})$, whilst the boundary condition is represented by

$$
\frac{\partial c}{\partial \mathbf{n}}=-\frac{c \mathbf{v} \cdot \mathbf{n}}{D} \quad \text { on } \partial \Omega(t)
$$

where $\mathbf{v}(\mathbf{x}, t)$ is the domain velocity, and $\mathbf{n}$ is the unit outward normal.

The arbitrary Lagrangian-Eulerian is a reference frame that combines features of the traditional Lagrangian and Eulerian frames. The Lagrangian frame, often used in solid-structure problems, corresponds directly to points within the structure and deforms with it. The Eulerian frame, meanwhile, is fixed in space, and allows average values of fluid passing a given region to be easily calculated. The ALE frame, meanwhile, is an arbitrary reference frame, able to move in space whilst permitting the flow of particles through the mesh (Belytschko and Kennedy 1978; Hughes et al. 1981; Donea et al. 1982).

Following Nobile (2001), we adopt a weak form of the ALE formulation of (6). We allow the movement of cells, which takes place at speed $\mathbf{w}(\mathbf{x}, t)$, to define the ALE frame. In this model the diffusing morphogen is external to the cells, and is not affected by their movement. Frameworks in which cells ingest the diffusing chemical, leading to advection terms in the governing equations, will be considered in future work. The problem can be stated as follows: find $c \in H^{1}(\Omega(t)):=\left\{y: \int_{\Omega(t)}\right.$ $\left.\left(|\nabla y|^{2}+y^{2}\right) \mathrm{d} \mathbf{x}<\infty\right\}$ such that for any test function $v \in H^{1}(\Omega(t))$

$$
\frac{\mathrm{d}}{\mathrm{d} t} \int_{\hat{\Omega}} \hat{v} \hat{c}\left|J_{t}\right| \mathrm{d} \hat{\mathbf{x}}+\int_{\Omega(t)} \nabla v \cdot(c \mathbf{w}) \mathrm{d} \mathbf{x}+\int_{\Omega(t)} \nabla v \cdot(D \nabla c) \mathrm{d} \mathbf{x}=\int_{\Omega(t)} v f \mathrm{~d} \mathbf{x}
$$

where $\hat{\Omega}$ is a static reference frame, with $\hat{\mathbf{x}}$ a point on this reference domain and concentration denoted by $\hat{c}(\hat{\mathbf{x}}, t) . J_{t}$ is the Jacobian of the mapping from $\Omega(t)$ to $\hat{\Omega}$. The test functions in the static frame are denoted $\hat{v}$. 


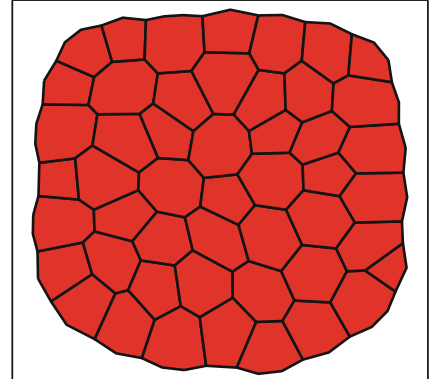

(a)

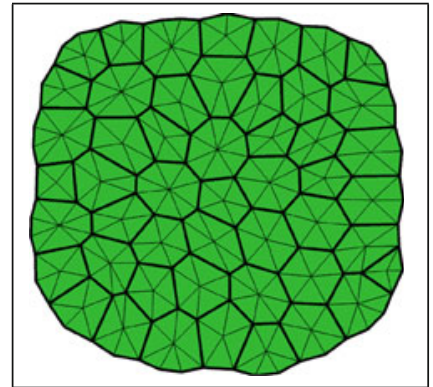

(b)

Fig. 4 a A cell configuration and $\mathbf{b}$ its counterpart FE mesh. The center of each cell is used as an additional node-point along with the cell vertices to create a triangular tessellation of the domain

\subsection{Fully discrete approximation}

We replace the continuous infinite-dimensional problem (7) with a discrete finitedimensional approximation. A set of $N(t)$ node-points $\left\{\mathbf{y}_{i}\right\}_{i=1}^{N(t)}$ in $\Omega(t)$ are connected to form a mesh of the domain consisting of $M(t)$ triangular elements $\left\{\tau_{i}\right\}_{i=1}^{M(t)}$. The triangles are such that $\operatorname{int}\left(\cup_{i} \bar{\tau}_{i}\right)=\Omega(t)$ and $\bar{\tau}_{i} \cap \bar{\tau}_{j}$ for $i \neq j$ is either a node, an edge, or is empty. We define $V_{h}(\Omega(t))$ to be a finite-dimensional subspace of $H^{1}(\Omega(t))$, consisting of continuous functions that are linear within each element $\tau_{i}$. A basis of $V_{h}(\Omega(t))$ is given by the functions $\left\{\phi_{i}(\mathbf{x}, t)\right\}_{i=1}^{N(t)}$, where for each $i, \phi_{i}$ is a continuous piecewise linear function such that $\phi_{i}\left(\mathbf{y}_{j}, t\right)=\delta_{i j}$ (the Kronecker delta function). To create the triangular FE mesh, we take the pre-existing cell vertices, and use the center of each cell as an additional node-point. This is demonstrated in Fig. 4, which shows an original vertex 'mesh' (a) and its corresponding triangular FE mesh (b).

The FE approximation to $c(\mathbf{x}, t)$, denoted $c_{h}(\mathbf{x}, t)$, is defined by

$$
c_{h}(\mathbf{x}, t)=\sum_{i=1}^{N(t)} \alpha_{i}(t) \phi_{i}(\mathbf{x}, t)
$$

where $c_{h}(\mathbf{x}, t) \in V_{h}(\Omega(t))$, and $\alpha_{i}(t)$ is a real number that weights each basis function within the approximation.

We now define a discretisation in time over the range $(0, T]$ to match that of the vertex model (Sect. 2.4), given by $\cup_{n=1}^{n=N_{T}}\left(t^{n-1}, t^{n}\right]$, where $T$ is the final time, $N_{T}$ is the number of time-points, and $t^{n}-t^{n-1}=\Delta t$ for all $n$. The time-discrete FE approximation to $c(\mathbf{x}, t)$ is given by

$$
c_{h}^{n}(\mathbf{x})=\sum_{i=1}^{N^{n}} \alpha_{i}^{n} \phi_{i}^{n}(\mathbf{x}) .
$$


Integrating the first term of (7) in time between $t^{n-1}$ and $t^{n}$ yields

$$
\int_{t^{n-1}}^{t^{n}}\left(\frac{\mathrm{d}}{\mathrm{d} t} \int_{\hat{\Omega}} \hat{v} \hat{c}\left|J_{t}\right| \mathrm{d} \hat{\mathbf{x}}\right) \mathrm{d} t=\int_{\Omega^{n}} v^{n} c^{n} \mathrm{~d} \mathbf{x}-\int_{\Omega^{n-1}} v^{n-1} c^{n-1} \mathrm{~d} \mathbf{x},
$$

where a superscript $n$ on a function indicates that it is evaluated at $t=t^{n}$. Approximating the remaining terms in (7) as piecewise constant in each interval $\left(t^{n-1}, t^{n}\right]$ and integrating over this time range, we obtain the fully discrete weak form

$$
\begin{aligned}
& \sum_{i=1}^{N^{n}} \alpha_{i}^{n}\left(\int_{\Omega^{n}} v^{n} \phi_{i}^{n} \mathrm{~d} \mathbf{x}+\Delta t \int_{\Omega^{n}} \nabla v^{n} \cdot\left(D^{n} \nabla \phi_{i}^{n}\right) \mathrm{d} \mathbf{x}+\Delta t \int_{\Omega^{n}} \nabla v^{n} \cdot\left(\phi_{i}^{n} \mathbf{w}^{n}\right) \mathrm{d} \mathbf{x}\right) \\
& \quad=\Delta t \int_{\Omega^{n}} v^{n} f^{n} \mathrm{~d} \mathbf{x}+\sum_{i=1}^{N^{n-1}} \alpha_{i}^{n-1} \int_{\Omega^{n-1}} v^{n-1} \phi_{i}^{n-1} \mathrm{~d} \mathbf{x} .
\end{aligned}
$$

The left-hand side can be written as a row vector, containing the term within the outer brackets evaluated for each $\phi_{i}$, multiplied by a column of the $\alpha_{i}$ values. The right-hand side is a single number. To solve this problem at each time point $n$ for $N^{n}$ unknown $\alpha_{i}^{n}$ values, we must substitute in $N^{n}$ test functions $v^{n}$. The most obvious choice is to use the basis functions $\left\{\phi_{j}^{n}\right\}_{j=1}^{N^{n}}$. A series of rows is obtained, each using a different $\phi_{j}$, multiplied by the column vector of $\alpha_{i}$ values. The system can thus be written as a matrix equation of the form

$$
\left(L^{n}+W^{n}\right) \alpha^{n}=R^{n},
$$

where $L^{n}$ and $W^{n}$ are $N^{n}$ x $N^{n}$ matrices, while $\alpha^{n}$ and $R^{n}$ are $N^{n}$ x 1 column vectors. The elements in each matrix are given by

$$
\begin{aligned}
L_{j i}^{n} & =\int_{\Omega^{n}}\left(\phi_{j}^{n} \phi_{i}^{n}+\Delta t \nabla \phi_{j}^{n} \cdot\left(D^{n} \nabla \phi_{i}^{n}\right)\right) \mathrm{d} \mathbf{x}, \\
W_{j i}^{n} & =\Delta t \int_{\Omega^{n}} \nabla \phi_{j}^{n} \cdot\left(\phi_{i}^{n} \mathbf{w}^{n}\right) \mathrm{d} \mathbf{x}, \\
R_{j}^{n} & =\Delta t \int_{\Omega^{n}} \phi_{j}^{n} f^{n} \mathrm{~d} \mathbf{x}+\sum_{i=1}^{N^{n-1}} \alpha_{i}^{n-1} \int_{\Omega^{n-1}} \phi_{j}^{n-1} \phi_{i}^{n-1} \mathrm{~d} \mathbf{x} .
\end{aligned}
$$

\section{Convergence properties of solution}

Simulations of the morphogen spreading model can now be run, using Matlab's backslash operator to solve the system of matrix equations (9). We have not yet discussed the effect on the FE solution of T1 transitions or proliferation, and this will be 
dealt with in Sect. 6. Before moving on, however, we test the convergence properties of the FE approximation by comparing it to a more accurate solution. Since it is not possible to obtain analytical solutions for such complex systems, we choose to compare the coarse mesh approximation to a highly refined (in time and space) reference solution. This comparison is made by considering the error function (11)

$$
\left(\int_{0}^{T} \int_{\Omega(t)}\left|c(\mathbf{x}, t)-c^{\prime}(\mathbf{x}, t)\right|^{2} \mathrm{~d} \mathbf{x} \mathrm{d} t\right)^{1 / 2}
$$

where $c(\mathbf{x}, t)$ is the approximation and $c^{\prime}(\mathbf{x}, t)$ is the accurate numerical solution.

The FE mesh shown in Fig. 4b can be refined by sub-partitioning each triangle. New nodes are added at the mid-point of each triangle edge and joined to construct four smaller similar triangles. This process can be repeated ad infinitum, creating a highly refined mesh. By also using a small value of the time-step, $\Delta t$, we can find the accurate numerical solution with which other approximations can be compared.

In order to run these simulations, an initial configuration of cells must first be created. This is achieved using the Voronoi tessellation of a series of $2 \mathrm{D}$ points. The points are chosen in such a way that the initial width of the tissue is approximately one, and most cells are hexagons. The initial average area of each cell is given by $\sim 1 / N_{c}$, where $N_{c}$ is the total number of cells, and the initial average cell width is $\sim 1 / \sqrt{N_{c}}$, assuming the overall shape of the configuration is roughly a square. To assign initial volumes, it is assumed that the cells are roughly cuboidal, and we take mean volume $\propto$ mean(cell area $)^{3 / 2}$.

In these simulations a random initial cellular configuration of 100 cells relaxes to mechanical equilibrium. Each simulation is identical from the cellular point of view, with the various FE solutions superimposed. Simulations are kept as simple as possible at the cellular level, with no junctional rearrangements, cell growth, or proliferation allowed.

Setting $f=0$ in (6) yields the standard diffusion equation given by

$$
\frac{\partial c}{\partial t}=\nabla \cdot(D \nabla c) \text { in } \Omega(t)
$$

With no source or sink terms, the total quantity of morphogen in the system, $C_{\Omega}(t)$, approximated by

$$
C_{\Omega}(t)=\int_{\Omega(t)} c_{h} \mathrm{~d} \mathbf{x}=\int_{\Omega(t)} \sum_{i=1}^{N(t)} \alpha_{i} \phi_{i} \mathrm{~d} \mathbf{x}=\sum_{i=1}^{N(t)} \alpha_{i} \int_{\Omega(t)} \phi_{i} \mathrm{~d} \mathbf{x}
$$

remains constant for all $t$.

Simulations are run beginning with a step function for the chemical concentration that takes a value of one in a small strip at one edge of the tissue and zero everywhere 


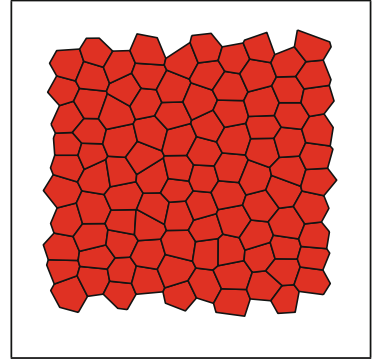

(a)

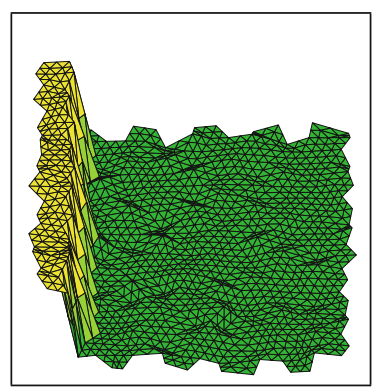

(d)

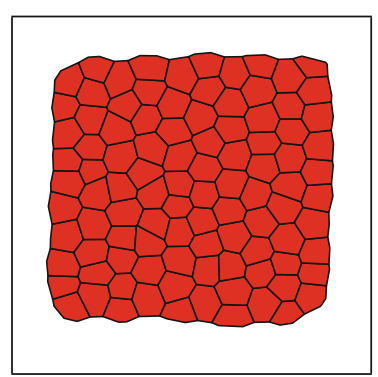

(b)

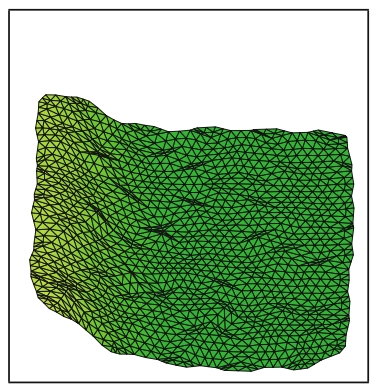

(e)

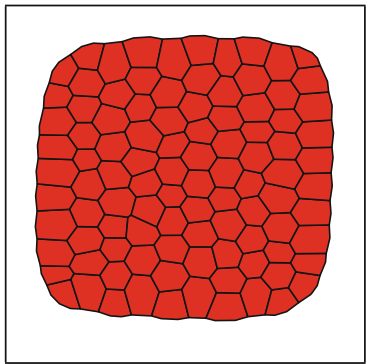

(c)

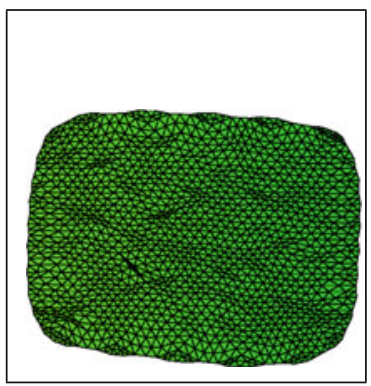

(f)

Fig. 5 a-c Initial random cellular configuration of 100 cells relaxing to mechanical equilibrium. d-f Chemical concentration, represented by height of the plot, diffusing on the same domain from an initial step function, with one iteration of mesh refinement. Images show $t=0(\mathbf{a}, \mathbf{d}), t=0.1(\mathbf{b}, \mathbf{e}), t=1(\mathbf{c}, \mathbf{f})$

Table 1 Parameter values used in simulations in this section

\begin{tabular}{llllll}
\hline Parameter & $C_{L}$ & $C_{P}$ & $C_{A}$ & $C_{H}$ & $C_{D}$ \\
Value & $2 \times 10^{-4}$ & $1 \times 10^{-4}$ & 1 & $1 \times 10^{-5}$ & $5 \times 10^{-4}$ \\
Parameter & $C_{B 1}$ & $C_{B 2}$ & $n_{1}-n_{4}$ & $\mu$ & $D$ \\
Value & $5 \times 10^{-3}$ & 0.1 & 2 & $1 \times 10^{-3}$ & 0.1 \\
\hline
\end{tabular}

All parameters, other than space- and time-steps for the FE solver, are the same in all simulations. See text for further details

else. The evolution of the tissue, as well as the concentration profile of the diffusing chemical, can then be observed over time (Fig. 5). Parameters used are given in Table 1. The relative values of the force constants are chosen such that each component has, on average, an approximately equal effect on each vertex. The time-steps, $\Delta t$, are sufficiently small that the movement of each vertex is small, relative to the average edge length, during each iteration.

We implement four test simulations, corresponding to four levels of mesh refinement, with the space- and time-steps each halving as the level number increases. The first level corresponds to no refinement of the mesh, i.e. the standard mesh shown in Fig. $4 \mathrm{~b}$, and a $\Delta t$ of $1 / 500$. The next levels correspond to one, two, and three refinements, with time-steps of 1/1000, 1/2000 and 1/4000, respectively. Each simulation 
Table 2 Value of the error function for each level of refinement. Space- and time-steps each halve as the refinement level increases

\begin{tabular}{ll}
\hline Refinement level & Error function \\
\hline 1 & $2.0 \times 10^{-4}$ \\
2 & $6.8 \times 10^{-5}$ \\
3 & $2.7 \times 10^{-5}$ \\
4 & $1.0 \times 10^{-5}$ \\
\hline
\end{tabular}

is run for $t \in(0,1]$. The accurate numerical solution is created with four levels of refinement, and a $\Delta t$ of $1 / 8000$. The value of the error function for each simulation is shown in Table 2.

We observe a clear convergence as the size of the space- and time-steps decrease. For each increase in refinement level, the error function decreases by a factor of between 2.5 and 2.9 .

\section{Simulation run time}

To get an idea of how simulation time of the numerical scheme scales with tissue size, we run three test simulations with tissues of 100 cells, 1000 cells, and 10,000 cells, respectively. The initial condition and parameter values are the same as in Sect. 4, and each simulation is run for 1000 iterations, corresponding to a $\Delta t$ of $1 / 1000$, with no refinement of the FE mesh. At each iteration, the time taken to form and solve the matrix equations is recorded, and we subsequently take the average time over the 1000 iterations.

Simulations are performed in Matlab, with matrices formed in $\mathrm{C}++$-based mex functions. The matrix system (9) is solved using the Matlab backslash operator. Simulations are performed on a 'no name' brand machine with 4 AMD (Advanced Micro Devices) Phenom(tm) II 945 processors (3GHz clock speed, 2MB L2 cache, 6MB L3 cache), 64-bit kernel running Ubuntu Linux 10.04 LTS. Results are shown in Table 3.

We observe that as the number of cells increases by a factor of 10 , the mean time taken per iteration increases by a factor between 12 and 15. For tissues with up to tens of thousands of cells it is therefore possible to accurately simulate in reasonable time-frames. These cell numbers correspond to most systems of interest biologically. We have not tested the scheme for much larger tissue sizes, of the orders of hundreds of thousands or millions of cells, which may show much larger increases in time as the cell number increases. These orders of magnitude may be relevant in some application areas, however in these cases continuum models might be more appropriate than the cell-based approach we take to modelling the tissue.

Table 3 Average time taken to form and solve the system of matrix equations for chemical concentration for tissues of 100 , 1000 , and 10,000 cells

\begin{tabular}{llll}
\hline Number of cells & 100 & 1000 & 10,000 \\
Number of nodes & 340 & 3130 & 30,400 \\
Number of elements & 600 & 6000 & 60,000 \\
Mean time per iteration (s) & $5.7 \times 10^{-3}$ & $6.7 \times 10^{-2}$ & 1.0 \\
\hline
\end{tabular}




\section{Effects of cellular rearrangements on the FE mesh}

\subsection{Proliferation}

As cell vertex positions are used to create the FE mesh, when a cell divides the mesh necessarily changes. The concentration of morphogen at the new vertices created by the division must therefore be found. Figure 6 shows the effect of mitosis on the mesh within the dividing cell from Sect. 2.6. The original mesh in the cell (a), consisting of six elements, changes to that shown in (b), with ten.

Since the FEM provides a continuous approximation in the spatial domain to the concentration $c(\mathbf{x}, t)$, we may interpolate to find the concentration at the two new nodes that are also cell vertices. For example, the concentration at node 9 can be calculated from the known concentrations at nodes 3 and 4 . If $d_{i j}$ represents the distance between nodes $i$ and $j$, then the concentration at node 9 is given by

$$
c_{9}=c_{3}+\frac{d_{39}}{d_{34}}\left(c_{4}-c_{3}\right) .
$$

These new vertices also affect the FE mesh within cells adjacent to the dividing cell that share the edges on which the new vertices have been created. For example, in this case the cells that share edges 1-6 and 3-4 will each gain an extra element. There are also two extra new nodes in the FE mesh at the centers of the new cells (10 and 11 in this example), while the node at the center of the previous cell (node 7), no longer exists.

The total concentration $C_{k}(t)$ in a cell $k$ is estimated by integrating the FE approximation to $c(\mathbf{x}, t)$ over the cell, i.e.

$$
C_{k}=\int_{k} c_{h} \mathrm{~d} \mathbf{x}=\int_{k} \sum_{l=1}^{L_{k}} \alpha_{l} \phi_{l} \mathrm{~d} \mathbf{x}=\sum_{l=1}^{L_{k}} \alpha_{l} \int_{k} \phi_{l} \mathrm{~d} \mathbf{x},
$$

where $l$ is a local node index and $L_{k}(t)$ is the number of nodes in cell $k$. Now, suppose at a given time-point $t=t^{n}$ the total concentration $C_{k}^{n}$ in a cell is known, along with

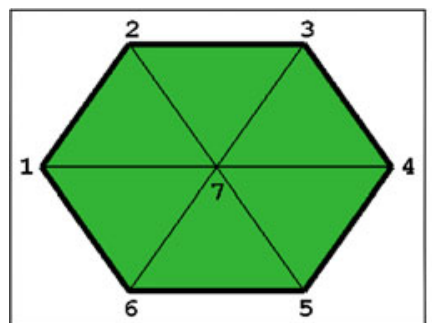

(a)

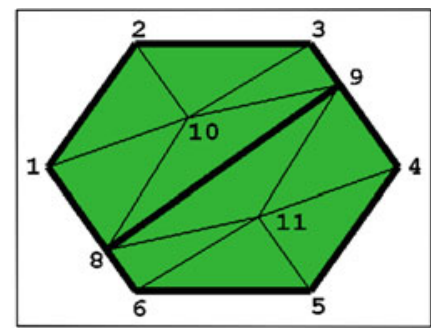

(b)

Fig. 6 Finite elements corresponding to hexagonal cell before and after mitosis. The cell in a contains six triangular elements, whilst the two daughter cells in $\mathbf{b}$ each contain five for a total of ten. The concentration at the four new nodes (8-11) must be calculated 


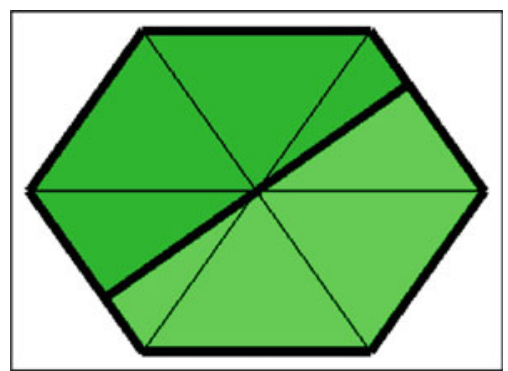

Fig. 7 Temporary FE mesh used during mitosis. This mesh contains the two new nodes that correspond to the two new cell vertices, but still has a node at the center of the mother cell, rather than the two new nodes at the centers of the two daughter cells

the values at all nodes apart from one. Assume without loss of generality that this is node $L_{k}^{n}$. The total concentration in cell $k$ at this time can be written

$$
C_{k}^{n}=\sum_{l=1}^{L_{k}^{n}-1} \alpha_{l}^{n} \int_{k} \phi_{l}^{n} \mathrm{~d} \mathbf{x}+\alpha_{L_{k}^{n}} \int_{k} \phi_{L_{k}^{n}} \mathrm{~d} \mathbf{x}
$$

and thus an expression for the value at node $L_{k}^{n}$ is obtained:

$$
\alpha_{L_{k}^{n}}=\frac{C_{k}^{n}-\sum_{l=1}^{L_{k}^{n}-1} \alpha_{l}^{n} \int_{k} \phi_{l}^{n} \mathrm{~d} \mathbf{x}}{\int_{k} \phi_{L_{k}^{n}} \mathrm{~d} \mathbf{x}} .
$$

Therefore, after division, if the total concentration in each of the new cells is known, the concentration value at the new central node can be found. The only remaining question is what the total concentration in each cell should be. The simplest method would be to find the total concentration in the mother cell and divide it equally between the two daughter cells. However, this neglects two important considerations. The first is that the areas of the daughter cells are not necessarily equal, and a larger cell is more likely to have a greater total concentration of morphogen. The second is that the cell could be sitting on a sharp gradient of morphogen, in which case the concentration in one section of the mother cell could be significantly greater than the rest of the cell, and this should be reflected in the daughter cells.

In order to solve this problem a temporary mesh for each of the new cells is adopted, as shown in Fig. 7, in order to calculate an approximation to the previous total concentration in the section of the mother cell now taken up by each daughter cell. This temporary mesh includes the two new cell vertices created during mitosis, but not the two new central nodes. The different shaded regions represent the two new cells. The values at all nodes are known, so (14) can be used to find the total concentration in each shaded region. Finally, we can apply (15) to find the values at the central nodes of the new cells. 


\section{2 $\mathrm{T} 1$ transitions}

T1 transitions also alter the FE mesh, though not as dramatically as proliferation events. In the example shown earlier in Fig. 2, cells 1 and 2 each lose an element, whereas cells 3 and 4 gain an extra element each. As this type of rearrangement only occurs on very short edges, the concentrations at nodes $\mathrm{C}$ and $\mathrm{D}$ are taken to be equal to the average of those at $\mathrm{A}$ and $\mathrm{B}$.

\section{Morphogen dependent growth}

In simulations to this point the diffusing chemical has been de-coupled from the mechanical properties of cells. By making cell growth explicitly dependent on morphogen concentration, we can begin to probe the role of the Dpp in the Drosophila imaginal wing disc, which has often been used as a model system for the study of morphogens. The disc grows from an embryonic primordium consisting of about 55 cells to reach a final size of roughly 30,500 (Martín et al. 2009). Growth is sigmoidal, with an average cell doubling time (CDT) of roughly 10-12 h (Garcia-Bellido and Merriam 1971), and is uniform across the disc.

Dpp is a morphogen that acts as an important growth-promoting factor in the wing disc. When Dpp expression is reduced, smaller wings are observed (Spencer et al. 1982), and large discs occur when Dpp is over-expressed (Burke and Basler 1996). The exact mechanism by which Dpp controls growth is unknown. One particular mystery is how a morphogen that forms a gradient can lead to uniform growth in the wing disc. Many theories have been postulated, none of which fully explain the observed phenomena (Serrano and O'Farrell 1997; Day and Lawrence 2000; Rogulja and Irvine 2005; Aegerter-Wilmsen et al. 2007; Hufnagel et al. 2007; Wartlick et al. 2011).

The framework developed in this paper can be used to test some of the hypotheses regarding the mechanisms by which Dpp controls growth. The first step towards this goal is to set up a steady gradient of Dpp across the cellular domain and allow the growth rate of cells to be dependent on the concentration of Dpp at the center of the cell. We expect proliferation to occur preferentially in regions of high Dpp concentration. We focus on this application in the rest of the paper. In order to examine growth regulation, changes to the application can be made to find those that elicit the desired uniform growth.

Equation (5) can be modified to be dependent on Dpp concentration as follows

$$
v_{k}^{n+1}=v_{k}^{n}\left(1+g_{k}\left(1+\lambda c_{k}\right) \Delta t\left(1-\frac{v_{k}^{n}}{V_{T}}\right)\right),
$$

where $c_{k}$ is the concentration of Dpp at the central node of cell $k$, and $\lambda$ is a parameter representing the strength of the effect of morphogen concentration on the growth rate. 


\subsection{Radial Dpp gradient}

A radial Dpp gradient can be set up by including source and degradation terms in system (11). Representing the source term as piecewise linear on the FEs, we can write

$$
f(\mathbf{x}, t)=\sum_{i=1}^{N(t)} f_{i}(t) \phi_{i}(\mathbf{x}, t)
$$

A subset of basis functions within a radius $r$ of the center of the tissue are designated to be source-producing, and their $f_{i}$ values set as follows:

$$
f_{i}=\left\{\begin{array}{l}
S\left|\mathbf{x}_{i}\right|<r \\
0\left|\mathbf{x}_{i}\right|>r
\end{array}\right.
$$

where $S$ is the magnitude of the source and $\mathbf{x}_{i}$ is the position of node $i$. Degradation of Dpp, meanwhile, is implemented by multiplying the final term of (8) by a constant $d$, with $0<d<1$. The equation for the vector $R$ in the matrix equations (11) therefore becomes

$$
R_{j}=\Delta t \sum_{i}^{N^{n}} f_{i}^{n} \int_{\Omega^{n}} \phi_{i}^{n} \phi_{j}^{n} \mathrm{~d} \mathbf{x}+d \sum_{i=1}^{N^{n-1}} \alpha_{i}^{n-1} \int_{\Omega^{n-1}} \phi_{j}^{n-1} \phi_{i}^{n-1} \mathrm{~d} \mathbf{x} .
$$

Figure 8 shows a sequence of images from a simulation, with parameter values given by Table 4 . In this simulation the final number of cells $N_{c}(t=T)=1011$.

As growth is made dependent on Dpp concentration, it is expected that cells in central regions will grow faster than those on the outside, and subsequently proliferate more frequently. However, as shown by Fig. 9a, this is not immediately clear from a histogram of mitosis locations in terms of distance from the center of the tissue. A correction must be made for the fact that the total number of cells in a region between radii $r$ and $r+d r$ for small $d r$ is approximately proportional to $r$. As there are therefore more cells at larger radii, there will be extra proliferation events, so a direct comparison is misleading. The histogram data are therefore divided by the radius at the center of each bin. As the tissue is growing, only cell divisions that occur within its original radius are considered. The relative number of divisions at greater radii will be small as cells only exist in these regions for part of the simulations. Figure $9 \mathrm{~b}$ shows the corrected histogram, with the trend for more divisions closer to the center of the disc clearly visible.

\subsection{Dpp produced in a central strip}

The FEM solver can be used to represent Dpp produced in a central strip of cells, thus setting up a gradient along the horizontal $(x)$ axis. This is achieved in a similar manner to (17), however in this case the function depends on distance along the axis rather 


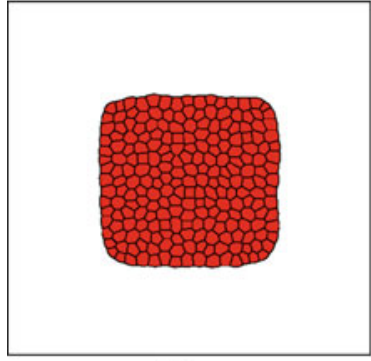

(a)

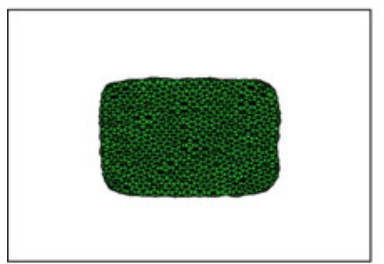

(d)

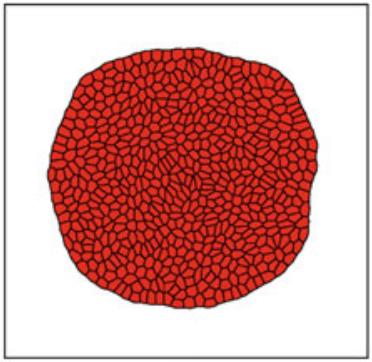

(b)

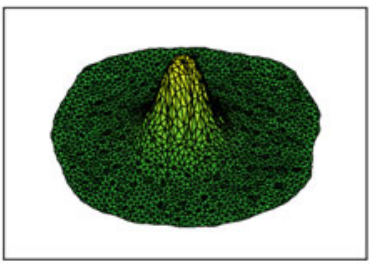

(e)

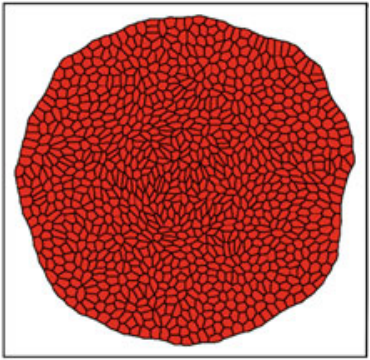

(c)

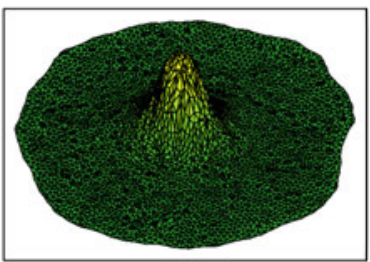

(f)

Fig. 8 Simulation of tissue growth with radial Dpp gradient. a-c The tissue evolves with proliferation and junctional rearrangements along with force laws. Cell growth is driven by the Dpp concentration in the cell. d-f The Dpp concentration is initially zero everywhere, before source is produced in a small radius around the center of the disc and diffuses throughout the domain

Table 4 Parameter values used in simulations of Drosophila wing disc

\begin{tabular}{lllllllll}
\hline Parameter & $N_{c}(t=0)$ & $C_{L}$ & $C_{P}$ & $C_{A}$ & $C_{H}$ & $C_{D}$ & $C_{B 1}$ & $C_{B 2}$ \\
Value & 225 & $2 \times 10^{-4}$ & $1 \times 10^{-4}$ & 10 & $5 \times 10^{-8}$ & $1 \times 10^{-4}$ & $1 \times 10^{-3}$ & 0.1 \\
Parameter & $n_{1}-n_{4}$ & $\mu$ & $\Delta t$ & $\lambda$ & $D$ & $S$ & $r$ & $d$ \\
Value & 2 & 1 & 1 & 2 & $1 \times 10^{-4}$ & 0.01 & 0.15 & 0.99 \\
\hline
\end{tabular}
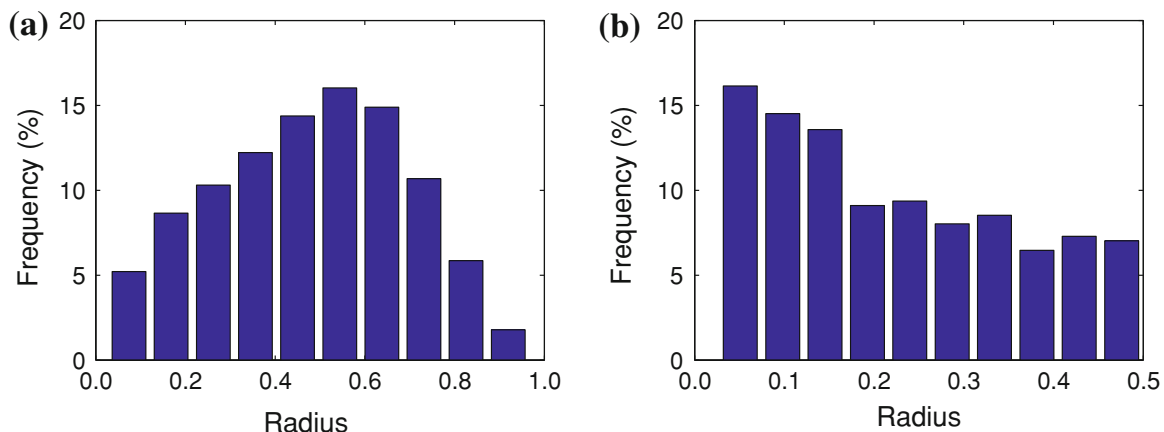

Fig. 9 Histograms of mitosis locations for simulation of tissue growth with radial Dpp gradient. a Mitosis locations in terms of distance from center of tissue. b Mitosis locations corrected for total number of cells at each radius 


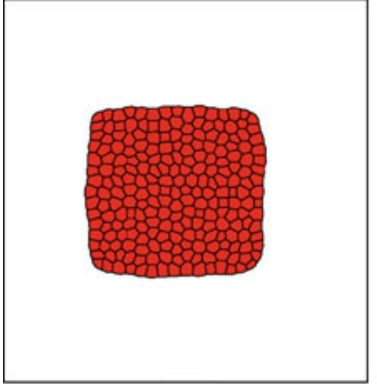

(a)

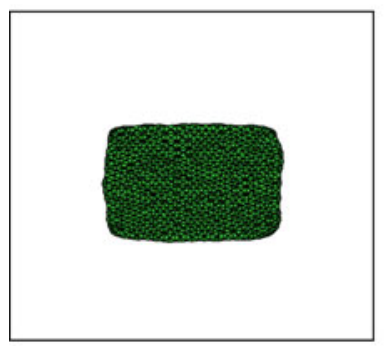

(d)

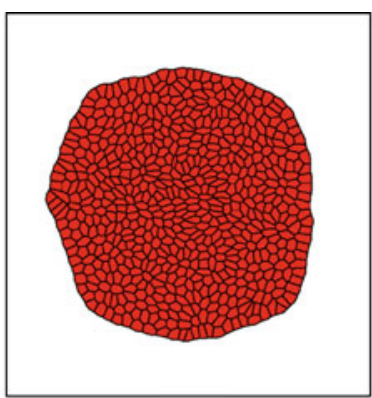

(b)

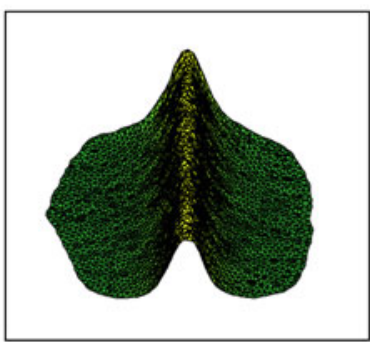

(e)

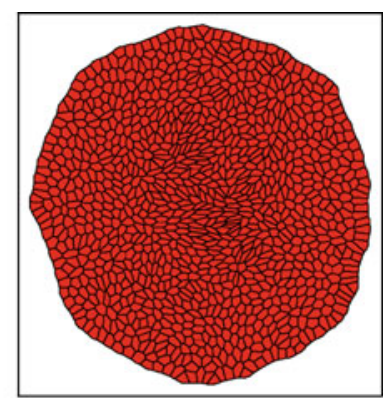

(c)

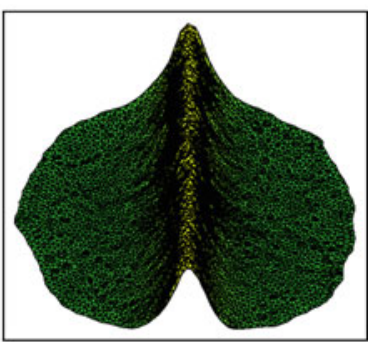

(f)

Fig. 10 Simulation of tissue growth with Dpp produced in central strip. a-c The tissue grows through time by Dpp-driven cell growth and subsequent proliferation. $\mathbf{d}-\mathbf{f}$ The Dpp concentration is initially zero everywhere, before source is produced in a small strip in the center of the disc and diffuses throughout the domain

than radial distance from the center of the tissue. As before in (16), growth is made dependent on the concentration of Dpp. The parameters used in this simulation are the same as those in Table 4. In this case $N_{c}(t=T)=1129$. Figure 10 shows that the tissue then grows inhomogeneously, with greater growth in the central regions. The tissue is elongated along the vertical axis in comparison with the horizontal, as cells are pushed vertically due to the excess proliferation near the central source of morphogen. This effect can also be observed by plotting the $x y$-ratio, a measure of the maximum width in the $x$-axis over the maximum height in the $y$-axis (Fig. 11).

This elongation of the tissue is a consequence of the growth rate of cells being dependent on the Dpp concentration, which is greater in these regions. As cells are growing faster in this region they reach the target volume more quickly, and subsequently divide more often. A histogram of mitosis locations along the $x$-axis shows a distribution that peaks in the central regions, as expected (Fig. 12a). However, it should be expected that proliferation is roughly even in the outer regions, and this is not the case. The reason for this is that it must again be considered that there are more total cells centrally, so mitosis is more likely to occur here anyway. Considering a circle centered at the origin, at a given $x$-value, the length of a vertical chord is $2\left(r^{2}-x^{2}\right)^{1 / 2}$. For a circular tissue the number of cells at a given $x$-value is proportional to this length, so the histogram data must be divided by this number to obtain the corrected values (Fig. 12b). Only mitosis events within the original radius of the 


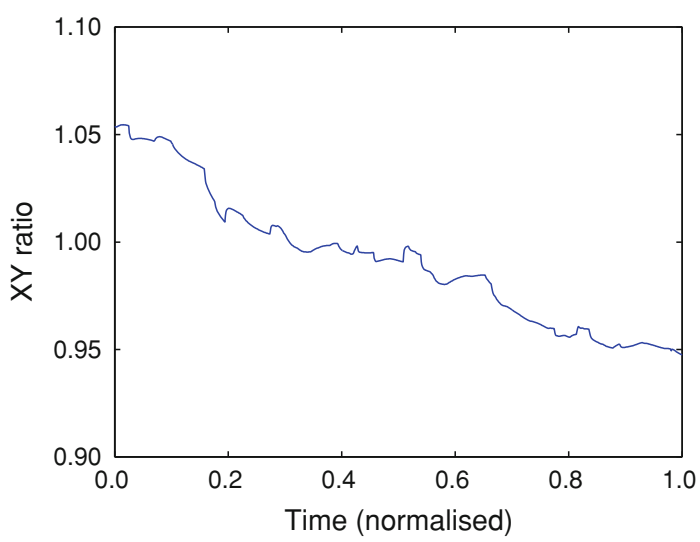

Fig. 11 Plot of $x y$-ratio for tissue growing under the influence of Dpp produced in a central strip. The $x y$-ratio represents the ratio of the width to the height of the tissue. The $x y$-ratio decreases over the simulation as growth is faster in a central strip of vertical cells and thus elongates in the $y$-axis
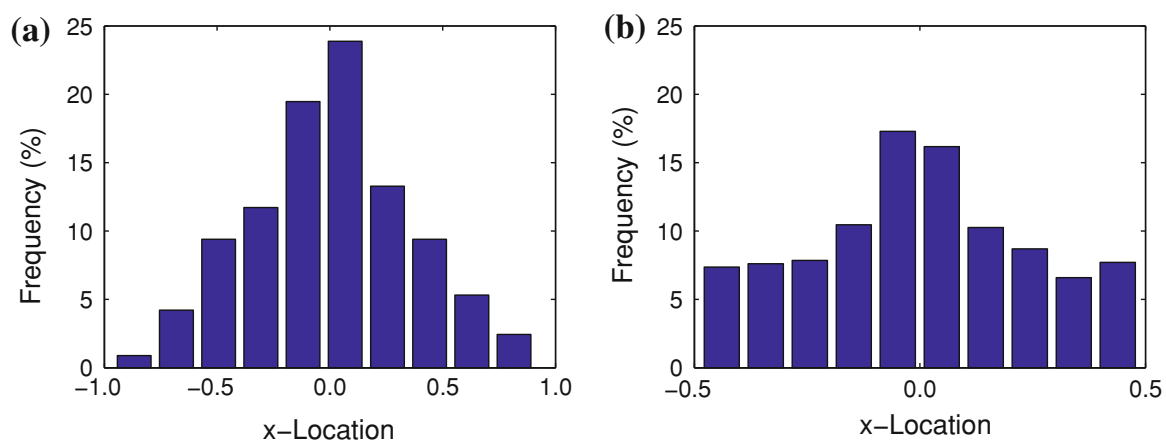

Fig. 12 Histograms of mitosis locations for simulation of tissue growth with Dpp produced in a central strip. a Mitosis locations along the $x$-axis. b Mitosis locations corrected for total number of cells at each $x$-value

tissue are considered, as explained in the previous example. The corrected distribution shows that cell division is roughly uniform in the outer regions, with a peak near the central strip, consistent with expectations.

In vivo, growth across the Drosophila imaginal wing disc is uniform. More complex examples are therefore required to shed insight into mechanisms that control growth. Suggested hypotheses include threshold models, where growth and proliferation are stimulated above a certain minimum concentration; gradient models, where cells respond to the local steepness of the Dpp gradient; mechanical feedback models, where stress and stretching as well as morphogen concentrations feedback into growth; and multiple-gradient models, where two or more morphogens combine to regulate growth. Our simulations have demonstrated that tissue-level effects can be elucidated by enabling a feedback between morphogen concentration and growth. Future work can focus on resolving which types of model faithfully replicate the observed phenomena. 


\section{Discussion}

A method for incorporating the dynamics of diffusing chemical substances within the framework of a vertex-based cell model has been described. In this type of model cells are represented as two-dimensional polygons, with forces acting on polygon vertices. Forces depend on quantities such as cell area, perimeter and deformation. Cells are also able to grow and divide and undergo basic junctional rearrangements. We have shown that by adopting an arbitrary Lagrangian-Eulerian formulation of diffusion-like equations, the FEM can be integrated smoothly into the vertex model to simulate morphogen spreading. Crucially, our set-up allows the chemical concentration to feedback into the vertex model and influence variables such as the growth rate of cells, the mechanical forces, or the angle of proliferation. This allows the relationship between mechanics and morphogens to be studied more accurately.

We implemented refinements of the spatial mesh and created an accurate numerical solution for the morphogen concentration in an example diffusion simulation. We defined an error function and showed that as space- and time-steps halve the error function decreases by a factor of between 2.5 and 2.9. We showed that the time taken to form and solve the system of matrix equations using Matlab's backslash operator scales well with cell number over orders of magnitude comparable to real systems of interest. Due to the size of the systems currently considered the backslash operator is a relatively fast and efficient solver. In future large-scale problems iterative solvers may need to be employed. In our simulations the time-step was chosen to be small due to the explicit nature of cell movement, not due to restrictions imposed by the numerical morphogen concentration solver. This is physically reasonable as the cell movements capture processes such as $\mathrm{T} 1$ transitions, which require precise knowledge of when the length of an edge falls below a certain threshold. In future work an adaptive scheme might be implemented, in which the time-step decreases if there are edge lengths in the system approaching the threshold, but can be much larger at other times.

We showed an example in which the growth rate of cells was made dependent on the concentration of a chemical representing Dpp in the Drosophila imaginal wing disc. It was shown that this led to a higher rate of proliferation in areas of the disc where Dpp chemical concentration was greatest. This example demonstrates a potential utility of the system, where simple hypotheses regarding the nature of growth factors can be tested to check if the results are consistent with what is known about growth of the tissue. Further work is required to decipher how uniform growth can result in the presence of a morphogen gradient. Our framework allows this kind of question to be examined systematically.

There are several possible extensions to the model that could be considered in future publications. The first of these is to incorporate cell death. This has been included in previous vertex models, and will also need to be dealt with in the morphogen model. We also hope to include a more sophisticated mesh refinement that can be implemented when necessary during simulations, based on the local deformation of the mesh, and can cope with the various rearrangement processes. Thirdly, cell surfaces contain receptors that bind with the diffusing chemical and lead to cellular ingestion. This process could be modelled, allowing cells to have an internal concentration that 
moves with the cell and affects its growth. This will require a system of two coupled equations, for the diffusing and internal chemical concentrations.

Our basic model is adaptable to many other systems. Dpp, which is just one example of a morphogen, is a crucial component in many developmental processes, in both Drosophila and other species. As simulations become more sophisticated, the potential for them to be adapted to solve problems in other areas will increase. It will also be possible to adjust the model to work with other types of cell representations. As long as the cell-domain can be broken up into elements for the FE mesh, and the movement of the domain is prescribed by rules between iterations, the formulation we propose can be implemented.

Acknowledgments AMS would like to thank EPSRC for funding via the Life Sciences Interface Doctoral Training Centre, University of Oxford. PKM was partially supported by a Royal Society Wolfson Research Merit Award.

\section{References}

Aegerter-Wilmsen T, Aegerter CM, Hafen E, Basler K (2007) Model for the regulation of size in the wing imaginal disc of Drosophila. Mech Dev 124(4):318-326

Aegerter-Wilmsen T, Smith AC, Christen AJ, Aegerter CM, Hafen E, Basler K (2010) Exploring the effects of mechanical feedback on epithelial topology. Development 137(3):499-506

Affolter M, Basler K (2007) The Decapentaplegic morphogen gradient: from pattern formation to growth regulation. Nat Rev Genet 8(9):663-674

Belytschko TB, Kennedy JM (1978) Computer models for subassembly simulation. Nucl Eng Des 49(1-2): 17-38

Bittig T, Wartlick O, Kicheva A, González-Gaitán M, Jülicher F (2008) Dynamics of anisotropic tissue growth. New J Phys 10(6):063001

Burke R, Basler K (1996) Dpp receptors are autonomously required for cell proliferation in the entire developing Drosophila wing. Development 122(7):2261-2269

Day SJ, Lawrence PA (2000) Measuring dimensions: the regulation of size and shape. Development 127(14):2977-2987

Donea J, Giuliani S, Halleux JP (1982) An arbitrary Lagrangian-Eulerian finite element method for transient dynamic fluid-structure interactions. Comput Method Appl Mech Eng 33(1-3):689-723

Farhadifar R, Röper J-C, Aigouy B, Eaton S, Jülicher F (2007) The influence of cell mechanics, cell-cell interactions, and proliferation on epithelial packing. Curr Biol 17(24):2095-2104

Garcia-Bellido A, Merriam JR (1971) Parameters of the wing imaginal disc development of Drosophila melanogaster. Dev Biol 24(1):61-87

Honda H (1978) Description of cellular patterns by Dirichlet domains: the two-dimensional case. J Theor Biol 72(3):523-543

Hufnagel L, Teleman AA, Rouault H, Cohen SM, Shraiman BI (2007) On the mechanism of wing size determination in fly development. Proc Natl Acad Sci USA 104(10):3835-3840

Hughes TJR, Kam Liu W, Zimmermann TK (1981) Lagrangian-Eulerian finite element formulation for incompressible viscous flows. Comput Method Appl Mech Eng 29(3):329-349

Kicheva A, Pantazis P, Bollenbach T, Kalaidzidis Y, Bittig T, Jülicher F, González-Gaitán M (2007) Kinetics of morphogen gradient formation. Science 315(5811):521-525

Landsberg KP, Farhadifar R, Ranft J, Umetsu D, Widmann TJ, Bittig T, Said A, Jülicher F, Dahmann C (2009) Increased cell bond tension governs cell sorting at the Drosophila anteroposterior compartment boundary. Curr Biol 19(22):1950-1955

Martín FA, Herrera SC, Morata G (2009) Cell competition, growth and size control in the Drosophila wing imaginal disc. Development 136(22):3747-3756

Nagai T, Honda H (2001) A dynamic cell model for the formation of epithelial tissue. Philos Mag B 81:699-719 
Nobile F (2001) Numerical approximation of fluid-structure interaction problems with application to haemodynamics. PhD thesis, École Polytechnique Fédérale de Lausanne, Switzerland

Odell GM, Oster G, Alberch P, Burnside B (1981) The mechanical basis of morphogenesis. Part I: epithelial folding and invagination. Dev Biol 85(2):446-462

Rauzi M, Verant P, Lecuit T, Lenne P-F (2008) Nature and anisotropy of cortical forces orienting Drosophila tissue morphogenesis. Nat Cell Biol 10(12):1401-1410

Rogulja D, Irvine KD (2005) Regulation of cell proliferation by a morphogen gradient. Cell 123(3): $449-461$

Serrano N, O'Farrell PH (1997) Limb morphogenesis: connections between patterning and growth. Curr Biol 7(3):R186-R195

Spencer FA, Hoffmann FM, Gelbart WM (1982) Decapentaplegic: a gene complex affecting morphogenesis in Drosophila melanogaster. Cell 28(3):451-461

Wartlick O, Mumcu P, Kicheva A, Bittig T, Seum C, Jülicher F, González-Gaitán M (2011) Dynamics of Dpp signaling and proliferation control. Science 331(6021):1154-1159

Weliky M, Oster G (1990) The mechanical basis of cell rearrangement. Part I: epithelial morphogenesis during Fundulus epiboly. Development 109(2):373-386 